\title{
Article \\ Comparative Study of Sargassum fusiforme Polysaccharides in Regulating Cecal and Fecal Microbiota of High-Fat Diet-Fed Mice
}

\author{
Bin Wei ${ }^{1,2,+} \mathbb{D}$, Qiao-Li Xu ${ }^{2,+}$, Bo Zhang ${ }^{2}$, Tao-Shun Zhou ${ }^{2}$, Song-Ze Ke ${ }^{2}$, Si-Jia Wang ${ }^{2,3}$, Bin Wu ${ }^{4}$, \\ Xue-Wei $\mathrm{Xu}^{1, *}$ and Hong Wang ${ }^{2, *(D)}$
}

1 Key Laboratory of Marine Ecosystem and Biogeochemistry, State Oceanic Administration \& Second Institute of Oceanography, Ministry of Natural Resources, Hangzhou 310012, China; binwei@zjut.edu.cn

2 College of Pharmaceutical Science \& Collaborative Innovation Center of Yangtze River Delta Region Green Pharmaceuticals, Zhejiang University of Technology, Hangzhou 310014, China; liujiahui0829@163.com (Q.-L.X.); zhangbo_1632021@163.com (B.Z.); zhoutaoshun@yeah.net (T.-S.Z.); kesongzeke@163.com (S.-Z.K.); new8090@hotmail.com (S.-J.W.)

3 Center for Human Nutrition, David Geffen School of Medicine, University of California, Rehabilitation Building 32-21, 1000 Veteran Avenue, Los Angeles, CA 90024, USA

4 Zhoushan Campus, Ocean College, Zhejiang University, Zhoushan 316021, China; wubin@zju.edu.cn

* Correspondence: xuxw@sio.org.cn (X.-W.X.); hongw@zjut.edu.cn (H.W.); Tel.: +86-571-8832-0622 (H.W.)

+ These authors contributed equally to this work.

Citation: Wei, B.; Xu, Q.-L.; Zhang, B.; Zhou, T.-S.; Ke, S.-Z.; Wang, S.-J.; Wu, B.; Xu, X.-W.; Wang, H. Comparative Study of Sargassum fusiforme Polysaccharides in Regulating Cecal and Fecal Microbiota of High-Fat Diet-Fed Mice. Mar. Drugs 2021, 19, 364. https:// doi.org/10.3390/md19070364

Academic Editor: Marc Diederich

Received: 25 May 2021

Accepted: 21 June 2021

Published: 24 June 2021

Publisher's Note: MDPI stays neutral with regard to jurisdictional claims in published maps and institutional affiliations.

Copyright: (c) 2021 by the authors. Licensee MDPI, Basel, Switzerland. This article is an open access article distributed under the terms and conditions of the Creative Commons Attribution (CC BY) license (https:/ / creativecommons.org/licenses/by/ $4.0 /)$.
Abstract: Seaweed polysaccharides represent a kind of novel gut microbiota regulator. The advantages and disadvantages of using cecal and fecal microbiota to represent gut microbiota have been discussed, but the regulatory effects of seaweed polysaccharides on cecal and fecal microbiota, which would benefit the study of seaweed polysaccharide-based gut microbiota regulator, have not been compared. Here, the effects of two Sargassum fusiforme polysaccharides prepared by water extraction (SfW) and acid extraction (SfA) on the cecal and fecal microbiota of high-fat diet (HFD) fed mice were investigated by $16 \mathrm{~S}$ rRNA gene sequencing. The results indicated that 16 weeks of HFD dramatically impaired the homeostasis of both the cecal and fecal microbiota, including the dominant phyla Bacteroidetes and Actinobacteria, and genera Coriobacteriaceae, S24-7, and Ruminococcus, but did not affect the relative abundance of Firmicutes, Clostridiales, Oscillospira, and Ruminococcaceae in cecal microbiota and the Simpson's index of fecal microbiota. Co-treatments with SfW and SfA exacerbated body weight gain and partially reversed HFD-induced alterations of Clostridiales and Ruminococcaceae. Moreover, the administration of SfW and SfA also altered the abundance of genes encoding monosaccharide-transporting ATPase, $\alpha$-galactosidase, $\beta$-fructofuranosidase, and $\beta$-glucosidase with the latter showing more significant potency. Our findings revealed the difference of cecal and fecal microbiota in HFD-fed mice and demonstrated that SfW and SfA could more significantly regulate the cecal microbiota and lay important foundations for the study of seaweed polysaccharide-based gut microbiota regulators.

Keywords: Sargassum fusiforme polysaccharides; high-fat diet; cecal microbiota; fecal microbiota; $16 \mathrm{~S}$ rRNA gene sequencing

\section{Introduction}

Gut microbiota is a population of microorganisms that colonizes the intestines. This not only protects against pathogens, provides nutrients, and maintains the integrity of the mucosal barrier, but also plays an important role in numerous diseases, such as inflammatory bowel disease, obesity, diabetes mellitus, metabolic syndrome, atherosclerosis, non-alcoholic fatty liver disease, etc. [1-4]. Owing to sampling difficulties, most studies chose to characterize the gut microbiota composition by sequencing the fecal samples, other than the cecal contents $[5,6]$. The differences of cecal microbiota and fecal microbiota in mice 
and human volunteers have been compared by several research groups [7-9]. Guo et al. reported that the relative abundance of an unidentified genus from the S24-7 family (S24-7) in cecal microbiota was much higher than that in fecal microbiota, and oral administration of a marine carotenoid, fucoxanthin, significantly increased the abundance of S24-7 in cecal microbiota but decreased the abundance of the genus in fecal microbiota [7]. Stanley et al. found that fecal and cecal microbiotas showed qualitative similarities but quantitative differences [8]. Marteau et al. compared the bacterial compositions within the human cecal and fecal microbiota and found that the abundance of Bifidobacteria, Bacteroides, Clostridium coccoides group, and Clostridium leptum subgroup were significantly lower in the cecum [9]. Therefore, comparative studies on the cecal and fecal microbiota in a specific situation are necessary to better understand the characteristics of gut microbiota.

High-fat diet (HFD) feeding has been widely used as a model for studying metabolic syndrome and gut microbiota dysbiosis $[10,11]$. Seaweed polysaccharides represent a kind of promising natural product that alleviates HFD-induced metabolic syndrome and promotes the healthy growth of gut bacteria [12]. In recent years, polysaccharides prepared from Sargassum fusiforme, a well-known edible alga, have attracted extensive research interest due to their potential biomedical application [13-17]. For example, our recent study indicated five polysaccharides prepared from $S$. fusiforme could selectively regulate the relative abundance of Oscillospira and Clostridiales in cecal microbiota of HFD-fed mice [16]. Cheng and colleagues prepared an S. fusiforme polysaccharide that could decrease the relative abundances of the diabetes-related fecal microbiota [17]. However, the regulatory effects of S. fusiforme polysaccharides on cecal microbiota and fecal microbiota, which would be helpful for the study of seaweed polysaccharide-based gut microbiota regulators, have not been compared.

In this study, two polysaccharides were prepared from S. fusiforme by water extraction (SfW) and acid extraction (SfA), and their chemical structures were characterized according to our recent report [16]. Then, the effects of 16 weeks of SfW and SfA administration on the cecal and fecal microbiota of HFD-fed mice were investigated.

\section{Results}

\subsection{Chemical Structures of Sargassum fusiforme Polysaccharides}

The physicochemical properties of the two $S$. fusiforme polysaccharides prepared by water extraction (SfW) and acid extraction (SfA) are shown in Table 1. The results indicated that the chemical structures of SfW and SfA were quite similar. For example, they had comparable contents of total sugar $(70.0 \%$ vs. $62.4 \%)$ and sulfate group ( $28.5 \%$ vs. $31.3 \%)$, and their average molecular weights were also very close. Moreover, the monosaccharide compositions of the two polysaccharides were very similar. Both of them were mainly composed of glucose, fucose, and galactose with small amounts of mannose, glucuronic acid, and xylose, but the detailed molar ratio had a slight difference. For example, the content of xylose in SfW was lower than that in SfA (0.14 vs. 0.03), while glucose was more abundant in SfA (1.05 vs. 1.13).

Table 1. Physicochemical properties of Sargassum fusiforme polysaccharides.

\begin{tabular}{cccccccccc}
\hline \multirow{2}{*}{ Samples } & \multirow{2}{*}{ Total Sugar (\%) } & \multirow{2}{*}{ Sulfate (\%) } & \multirow{2}{*}{ Mw (kDa) } & \multicolumn{4}{c}{ Monosaccharide (Molar Ratio) * } \\
\cline { 5 - 10 } & & & & Man & Glc A & Glc & Gal & Xyl & Fuc \\
\hline SfW & 70.0 & 28.5 & $166 / 5.9$ & 0.07 & 0.07 & 1.05 & 0.41 & 0.14 & 1 \\
SfA & 62.4 & 31.3 & $276 / 5.8$ & 0.05 & 0.06 & 1.13 & 0.38 & 0.03 & 1 \\
\hline
\end{tabular}

* Man, mannose; GlcA, glucuronic acid; Glc, glucose; Gal, galactose; Xyl, xylose; Fuc, fucose.

Here, ${ }^{1} \mathrm{H}$ NMR spectra of SfW and SfA are shown in Figure S1. The resonance signals of the two polysaccharides at 3.0-5.5 ppm were ascribed to the typical distribution of ${ }^{1} \mathrm{H}$ NMR signals of the polysaccharides [18]. The unresolved peaks at 5.3-5.5 ppm were assigned to the anomeric protons of $\alpha$-L-fucopyranosyl units [19]. The resonance signals of 
the two polysaccharides at 3.3-4.5 ppm were apportioned to the ring protons $\mathrm{H}-2$ to $\mathrm{H}-5$, but the pattern was different from each other, and the chemical shifts at 1.1 and $1.4 \mathrm{ppm}$ were assigned to methyl groups of fucose units [20]. In addition, due to the complex and heterogeneous structure of sulfated polysaccharides, the broadening and overlapping of ${ }^{1} \mathrm{H}$ NMR peaks makes it difficult to completely describe their structural characteristics.

\subsection{Effects of SfW and SfA on HFD-Induced Metabolic Disorders}

The effects of SfW and SfA on HFD-induced metabolic disorders were evaluated after 16 weeks of co-treatment with HFD and polysaccharides. As shown in Figure 1A, 16-week HFD feeding significantly increased the body weight of the mice compared to that of the blank group, and co-treatment with SfW and SfA exacerbated body weight gain. The fasting blood glucose level significantly increased after 4 weeks of HFD-feeding, and only treatment with SfW at the fourth week reversed the increase $(p<0.05)$ (Figure 1B). In OGTT, the blood glucose reached the maximal level at $30 \mathrm{~min}$ after the dextrose gavage, and the blood glucose level of the control group was significantly higher than that of the blank group $(p<0.001)$. The polysaccharides administration could not attenuate the HFD-induced glucose intolerance (Figure 1C). Treatments with SfW and SfA did not protect HFD-induced insulin resistance and epididymal fat weight gain (Figure 1D-F).

(A)
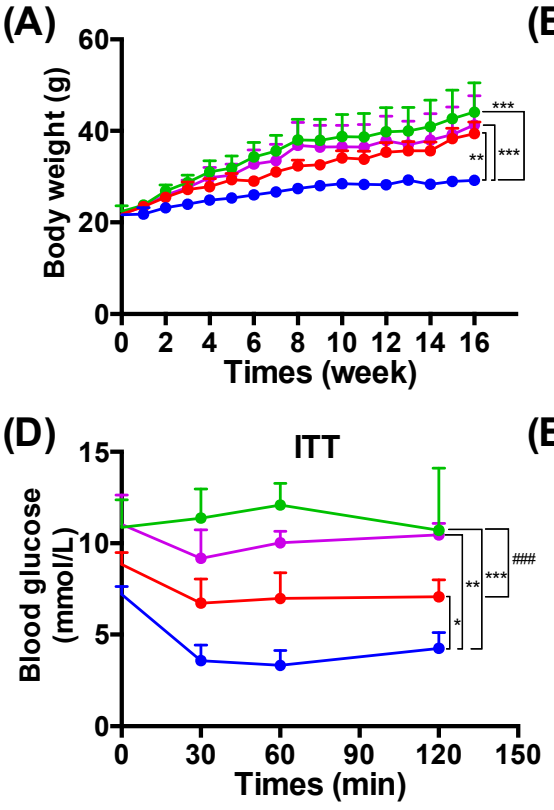

(B) :

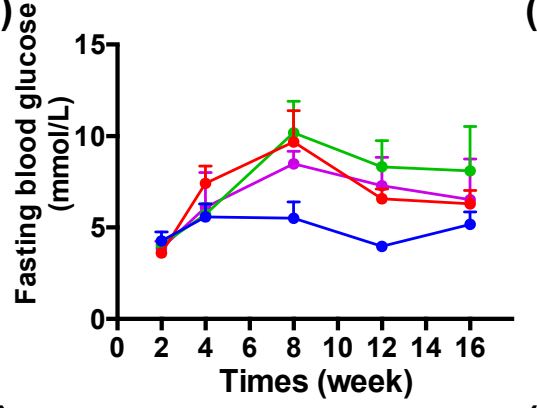

(E)

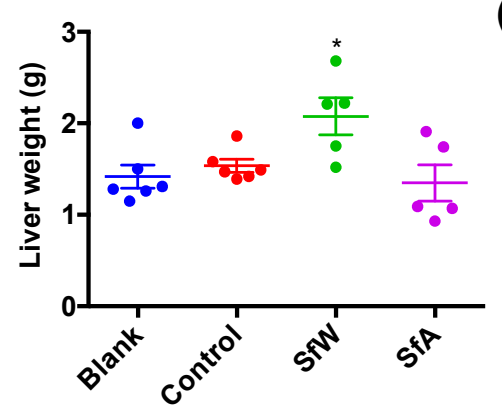

(C)

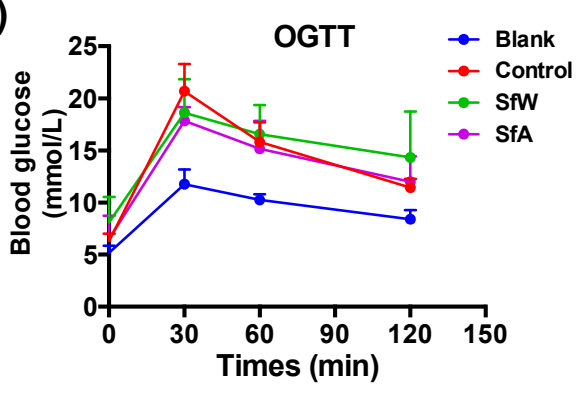

(F)

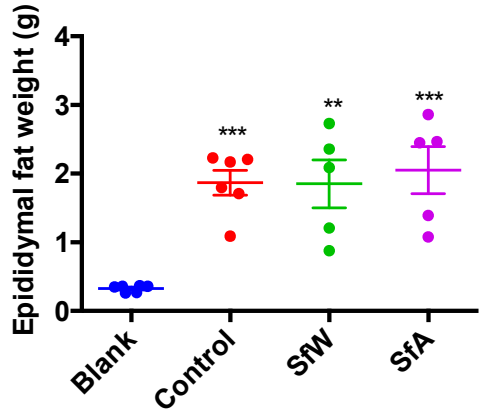

Figure 1. Effects of SfW and SfA on (A) body weight, (B) fasting blood glucose, (C) OGTT, (D) ITT, (E) liver weight, and (F) epididymal fat weight in HFD-fed mice. Values are mean $\pm \operatorname{SD}(n=5-6){ }^{*} p<0.05,{ }^{* *} p<0.01,{ }^{* * *} p<0.001$ vs. Blank. \#\#\# $p<0.001$ vs. Control. Data sets in (A-D) were analyzed using unpaired two-tailed Student's $t$-test. Data sets in (E-F) were analyzed using one-way ANOVA followed by a Turkey's test.

The effects of 16 weeks of HFD and polysaccharide administration on gut microbiota in fecal samples and cecal contents of mice were analyzed by 16S rRNA high-throughput sequencing. As shown in Figure 2A-B, HFD significantly decreased both the Chao1 and Simpson's indices of cecal microbiota but showed negligible effect on the Simpson's index of fecal microbiota. Oral administration of SfW and SfA did not significantly improve the decrease in $\alpha$-diversity of fecal and cecal microbiota. The unsupervised principal components analysis (PCA) plot at the phylum level showed that PC1 and PC2 were able to explain $56 \%$ and $41.3 \%$ of the variation, respectively, and exhibited significant distinction between the cecal and fecal microbiota of the blank group (Figure 2C). Polysaccharide administration showed no significant regulatory effect on the dysbiosis of cecal and fecal microbiota at the phylum level (Figure 2C). In detail, HFD significantly increased the 
relative abundance of Actinobacteria and decreased the abundance of Bacteroidetes and Verrucomicrobia in both the cecal and fecal microbiota but only enriched Firmicutes and Proteobacteria in fecal microbiota. SfW only presented a regulatory effect on Proteobacteria in fecal microbiota (Figure 2H,J).
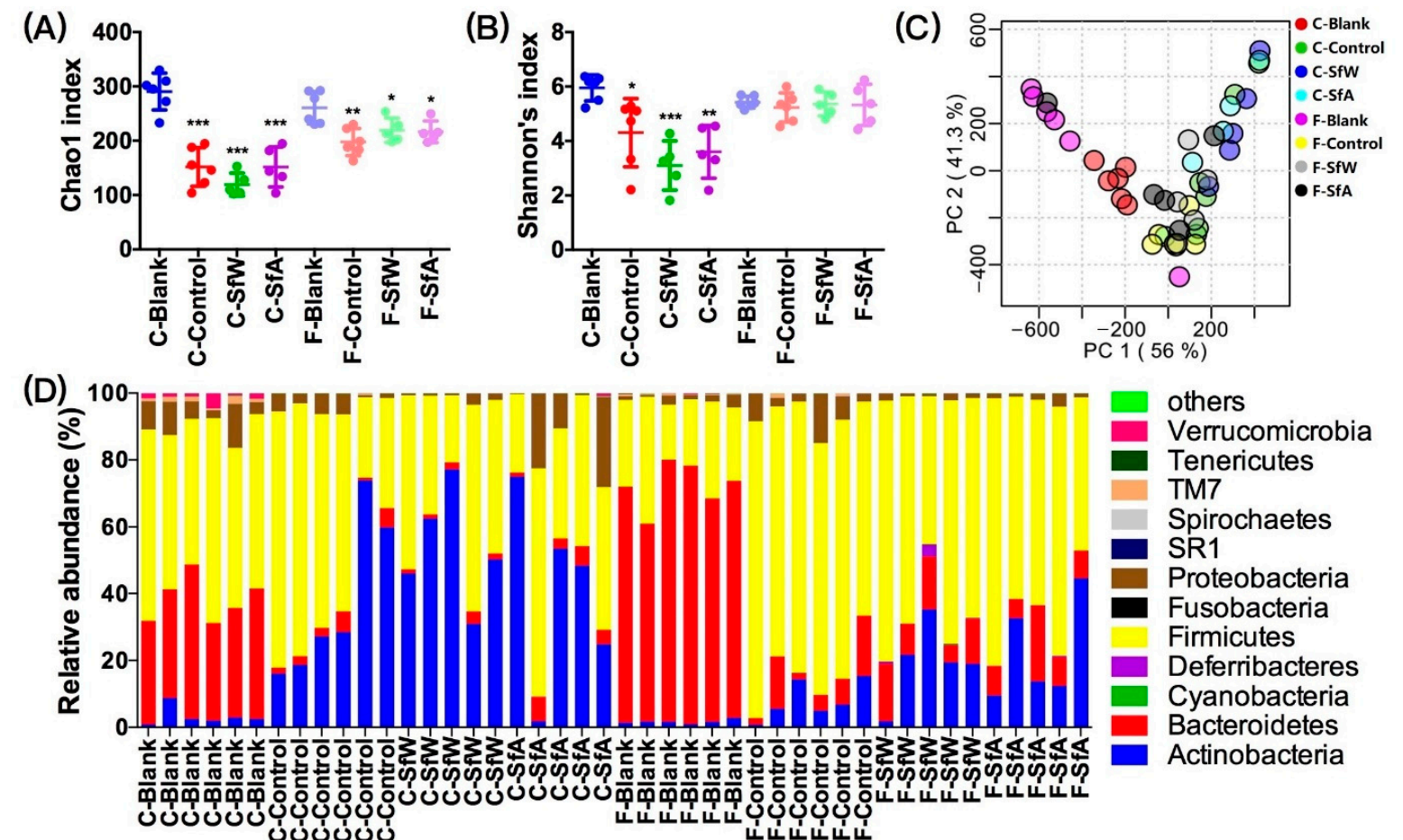

(E)

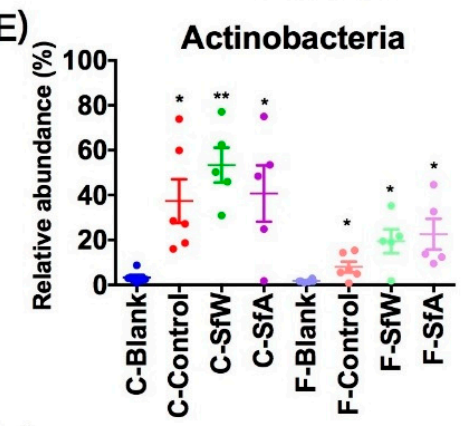

(H)

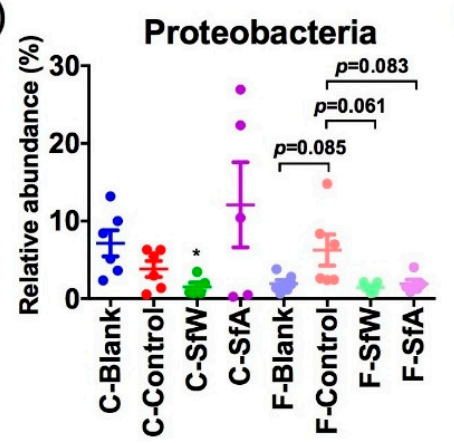

(F)

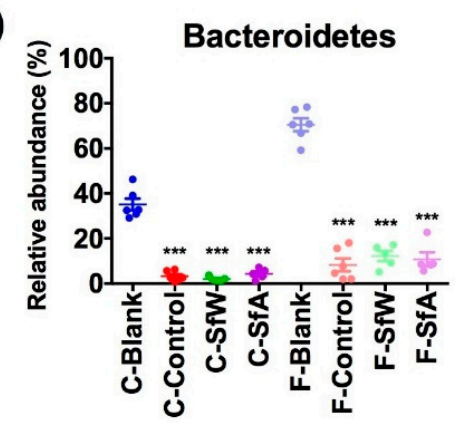

(I)

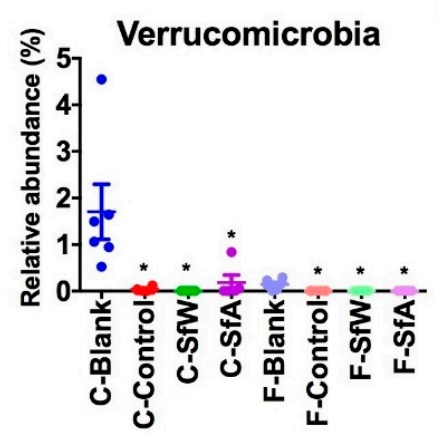

(G)

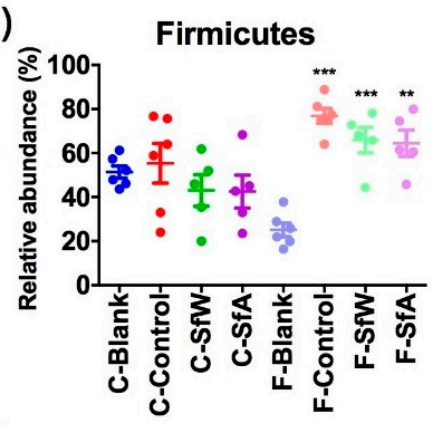

(J)

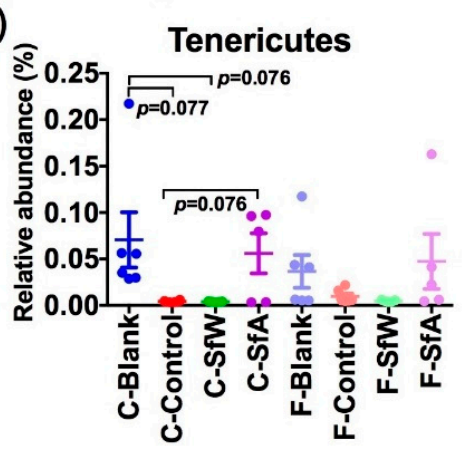

Figure 2. Effects of SfW and SfA on the (A) Chao1 diversity and (B) Shannon's diversity indices of cecal and fecal microbiota in HFD-fed mice. (C) PCA and (D) bar plots of cecal and fecal microbiota at the phylum level. The relative abundance of (E) Actinobacteria, (F) Bacteroidetes, (G) Firmicutes, (H) Proteobacteria, (I) Verrucomicrobia, and (J) Tenericutes in cecal and fecal microbiota. Values are mean \pm SEM. ${ }^{*} p<0.05,{ }^{* *} p<0.01,{ }^{* * *} p<0.001$ vs. the corresponding blank group. Data sets in (A), (B), (E-J) were analyzed using one-way ANOVA followed by a Turkey's test. C-, cecal microbiota; F-, fecal microbiota. 
The effects of SfW and SfA on the cecal and fecal microbiota in HFD-fed mice were also investigated at the genus level. As shown in Figure 3A, bacteria in these cecal and fecal samples mainly consisted of 27 genera, including Coriobacteriaceae and S24-7. However, the detailed composition of them between the cecal and fecal microbiota and between the blank and control groups were different. The far distance between the F-Blank group and F-SfW or F-SfA group in the clustering scheme suggested that HFD-induced dysbiosis of cecal microbiota could be more significantly regulated by SfW and SfA. The PCA plot at the genus level further confirmed the compositional difference between the cecal and fecal samples (Figure 3B). HFD mainly altered the relative abundance of Coriobacteriaceae, S24-7, Ruminococcus, Clostridiales, Oscillospira, Ruminococcaceae, and Akkermansia in both the cecal and fecal microbiota but only enriched Oscillospira in fecal microbiota (Figure 3C-I). Notably, oral administration of SfW and SfA could partially alleviate the increase of Clostridiales and Ruminococcaceae in fecal microbiota (Figure 3F,H).

(A)

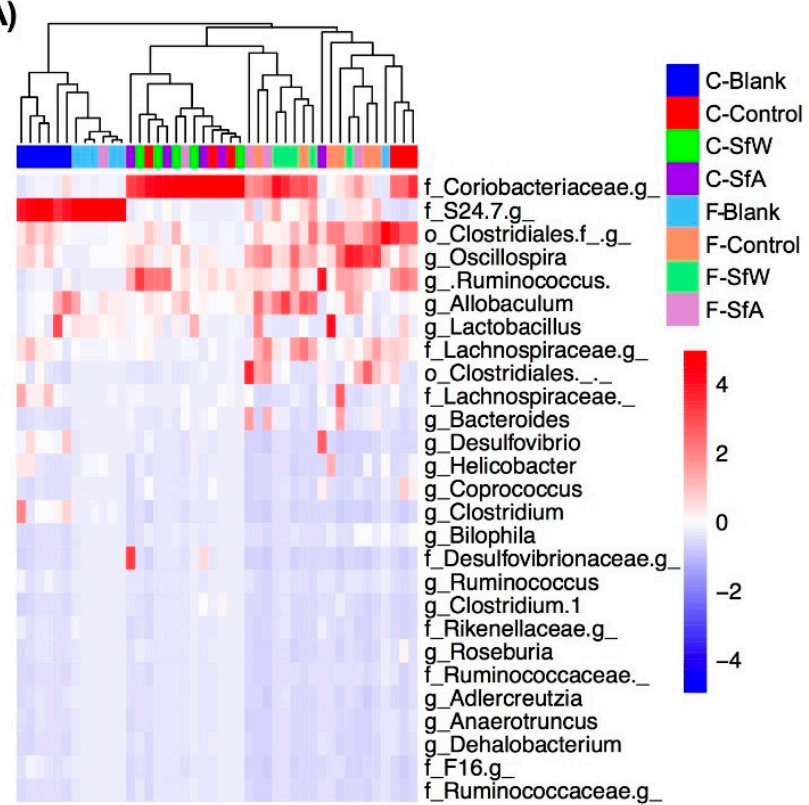

(F)

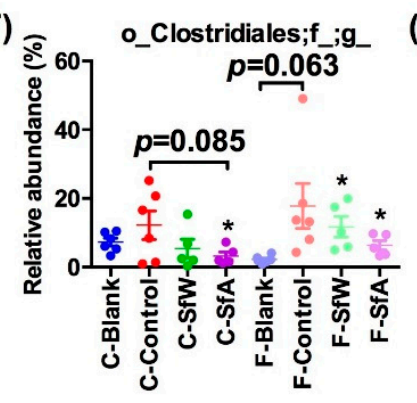

(B)

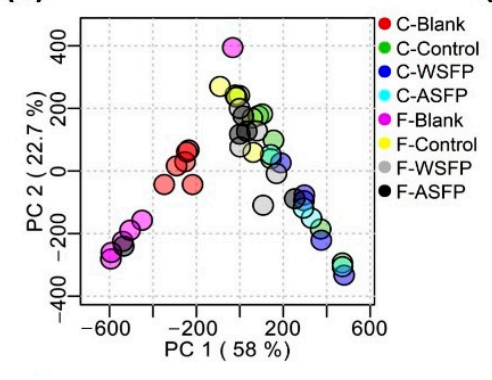

(D)

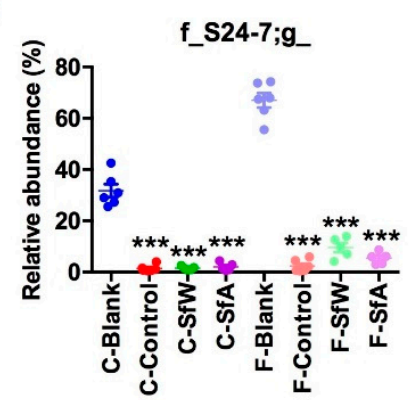

(H)

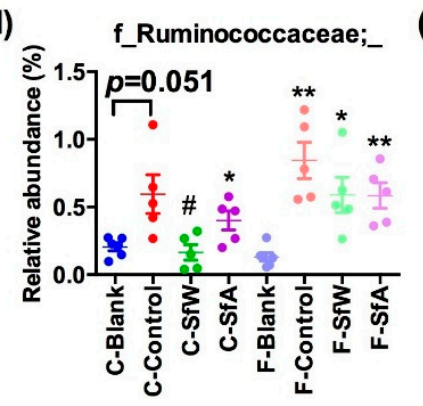

(C)

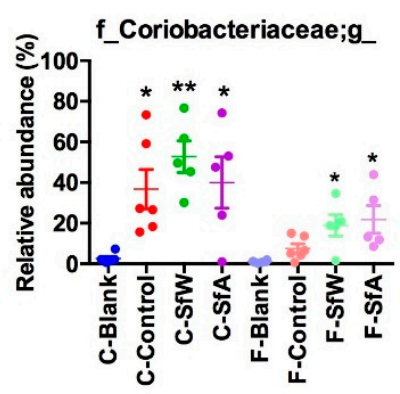

(E)
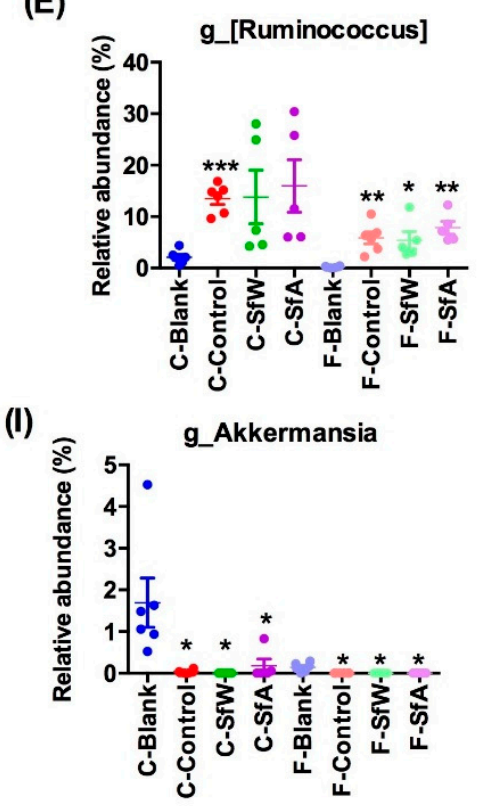

Figure 3. Effects of SfW and SfA on the cecal and fecal microbiota in HFD-fed mice. (A) Heatmap and (B) PCA plots of cecal and fecal microbiota at the genus level. The relative abundance of (C) Coriobacteriaceae, (D) S24-7, (E) Ruminococcus, (F) Clostridiales, (G) Oscillospira, (H) Ruminococcaceae, and (I) Akkermansia in cecal and fecal microbiota. Values are mean \pm SEM. ${ }^{*} p<0.05,{ }^{* *} p<0.01,{ }^{* * *} p<0.001$ vs. the corresponding blank group. $\# p<0.05$ vs. the corresponding control group. Data sets in (C-I) were analyzed using one-way ANOVA followed by a Turkey's test. C-, cecal microbiota; F-, fecal microbiota.

\subsection{Effects of SfW and SfA on the Abundance of Genes Encoding Carbohydrate-Metabolizing Enzymes in Cecal and Fecal Microbiota}

The effects of $S$. fusiforme polysaccharides on the abundance of genes encoding carbohydrate-metabolizing enzymes in the cecal and fecal microbiota of HFD-fed mice were investigated using PICRUSt2 based on the 16S rRNA gene sequencing data. The 
results demonstrated that the HFD significantly decreased the abundance of genes encoding $\alpha$-fucosidase (Figure 4A) and $\beta$-glucuronidase (Figure 4G), and increased that of monosaccharide-transporting ATPase (Figure 4B) and $\beta$-fructofur-anosidase (Figure $4 \mathrm{E}$ ) in both the cecal and fecal microbiota, but the alterations of genes encoding $\alpha$-galactosidase (Figure $4 \mathrm{D}$ ) and $\beta$-glucosidase (Figure $4 \mathrm{~F}$ ) were only observed in fecal microbiota, and that of $\beta$-mannosidase (Figure $4 \mathrm{H}$ ) was only presented in cecal microbiota. The administration of SfW and SfA mainly regulated the abundance of genes encoding monosaccharidetransporting ATPase, $\alpha$-galactosidase, $\beta$-fructofuranosidase, and $\beta$-glucosidase with the latter showed more significant potency. For example, SfA alleviated the increase of genes encoding monosaccharide-transporting ATPase and $\beta$-glucosidase (Figure $4 \mathrm{~B}-\mathrm{F}$ ).

(A)

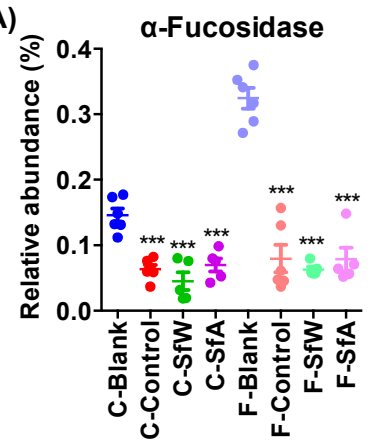

(E)

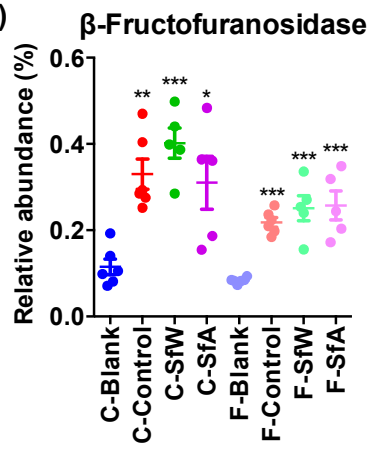

(B)

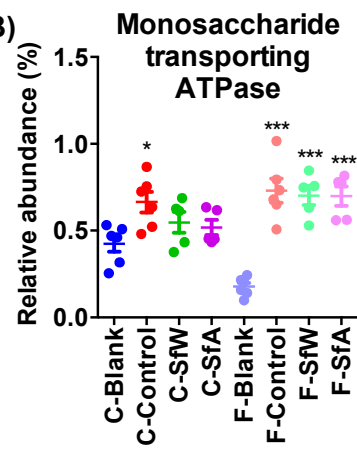

(F)

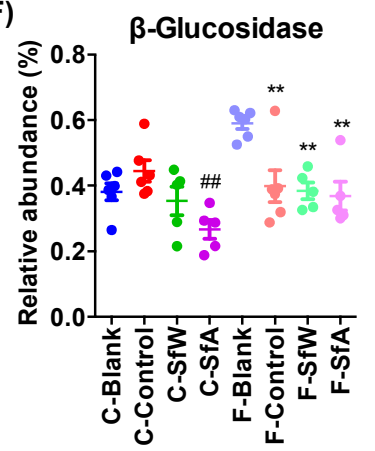

(C)

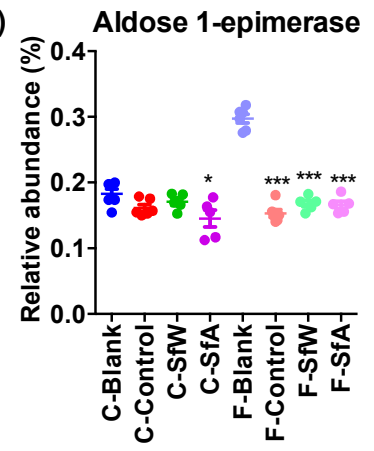

(G)

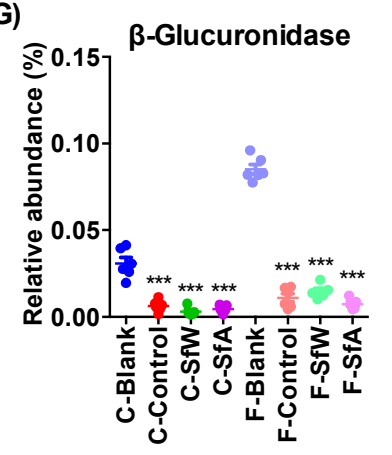

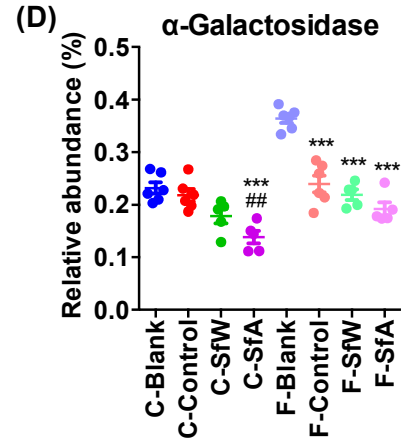

(H)

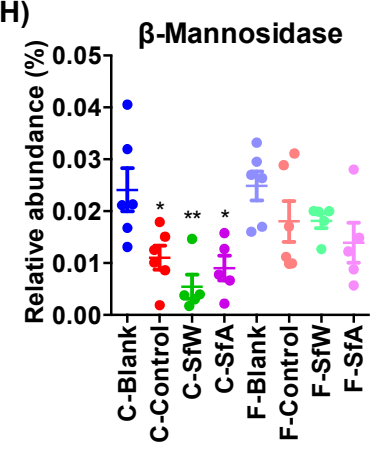

Figure 4. Effects of SfW and SfA on functional gene of cecal and fecal microbiota in HFD-fed mice. (A) $\alpha$-Fucosidase, (B) monosaccharide-transporting ATPase, (C) aldose 1-epimerase, (D) $\alpha$-galactosidase, (E) $\beta$-fructofuranosidase, (F) $\beta$ glucosidase, (G) $\beta$-glucuronidase, and (H) $\beta$-mannosidase. Values are mean \pm SEM. ${ }^{*} p<0.05,{ }^{* *} p<0.01,{ }^{* * *} p<0.001$ vs. the corresponding blank group. \#\# $p<0.01$ vs. the corresponding control group. Data sets were analyzed using one-way ANOVA followed by a Turkey's test. C-, cecal microbiota; F-, fecal microbiota.

\section{Discussion}

Seaweed polysaccharides have various biological activities $[11,13,21-25]$ and also show potential to be developed as prebiotics that promote the healthy growth of gut bacteria. Previous studies have compared the similarities and differences between the cecal and fecal microbiota of animals and human volunteers [6-8]. However, the regulatory effects of polysaccharides extracted from $S$. fusiforme (a common and widely eaten seaweed) on the cecal and fecal microbiota of HFD-fed mice, which would benefit the study of seaweed polysaccharide-based gut microbiota regulators, have not been compared. Here, the impacts of 16 weeks of water and acid extracted S. fusiforme polysaccharides (SfW and SfA) on the cecal and fecal microbiota of HFD-fed mice were investigated. We found that the HFD significantly altered the dominant phyla Bacteroidetes and Actinobacteria, and the dominant genera Coriobacteriaceae, S24-7, and Ruminococcus, but did not affect the abundance of Firmicutes, Clostridiales, Oscillospira, and Ruminococcaceae in cecal microbiota and the Simpson's index of fecal microbiota. Co-treatments with SfW and SfA partially reversed the dysbiosis of Firmicutes, S24-7, Ruminococcus, Clostridiales, and Ruminococcaceae. The administration of SfW and SfA also altered the abundance of some genes encoding 
carbohydrate-metabolizing enzymes between cecal and fecal microbiota in 16-week HFDfed mice and demonstrated that cecal microbiota was more significantly regulated by S. fusiforme polysaccharides.

Our recent study reported that five S. fusiforme polysaccharides prepared through hot-water and acid extraction showed a wide range of molecular weight (10-698.3 kDa), and the $\mathrm{HCl}$-extracted polysaccharide (Sf-A) mainly consisted of glucose, fucose, and galactose [16]. Cheng et al. prepared a $205.8 \mathrm{kDa}$ S. fusiforme polysaccharide (SFF) by acid extraction and the polysaccharide was mainly composed of fucose and galactose [17]. The chemical structures of SfW and SfA were more similar to Sf-A than SFF, which may be because they are sourced from the same supplier.

HFD has been demonstrated to adversely affect gut microbiota composition through increasing the abundance of Firmicutes and Proteobacteria and decreasing Bacteroidetes [26-29]. The present study demonstrated that 16 weeks of HFD significantly increased the relative abundance of Actinobacteria and decreased the abundance of Bacteroidetes and Verrucomicrobia in both the cecal and fecal microbiota but only enriched Firmicutes and Proteobacteria in fecal microbiota. The discrepancy between the previous studies with our findings indicates that HFD consistently decreases the abundance of Bacteroidetes in both the cecal and fecal microbiota, but the impacts on other phyla depend on the sampling position and the specific conditions of the animal models. Our recent study demonstrated that four weeks of HFD feeding significantly increased the relative abundance of Coriobacteriaceae and Oscillospira in cecal microbiota [11], but in the present study, the abundance of Oscillospira in cecal microbiota was not enriched by HFD, which may be explained by the prolonged HFD treatment. These findings further deepen our understanding about the impact of HFD on gut microbiota composition.

It is well known that microbiota composition varies in different parts of the gastrointestinal tract, and there are significant differences in the quantity and quality of microorganisms in the cecum contents and feces [8]. According to a recent report, the cecal and fecal microbiota exhibits different taxonomic structures, functional activities, and metabolic pathways [30]. Guo et al. also reported that the abundance of Firmicutes in the cecum of mice fed a normal diet was much higher than that in the feces, and S24-7 was much lower in the cecal contents, which was highly consistent with the present study [9]. Here, we comprehensively compared the similarities and differences between the cecal and fecal microbiota in 16-week HFD-fed mice, including the abundance of genes encoding carbohydrate-metabolizing enzymes in cecal and fecal microbiota. HFD showed a more significant influence on the $\alpha$-diversity of cecal microbiota than that of fecal microbiota, suggesting that cecal microbiota may be more suitable to represent the gut microbiota composition. Some bacteria (Actinobacteria and Coriobacteriaceae) were significantly enriched in the cecal contents, while others (Firmicutes, Oscillospira, and Ruminococcaceae) were enriched in the fecal samples, which may be associated with the different function of the cecum and colon. For example, Coriobacteriaceae, a family within the phylum Actinobacteria, are strictly anaerobic bacteria and contribute to the metabolism of bile salts, steroids, and dietary polyphenols [31]. Ariangela et al. reported that the TNBS colitis severity was most closely correlated with the composition of colonic mucus microbiome, but not fecal or cecal microbiome [32], suggesting that the choice of sampling site depends on the experimental design. Interestingly, SfA was mainly composed of glucose, fucose, and galactose, and administration of SfA significantly decreased the abundance of genes encoding $\alpha$-galactosidase and $\beta$-glucosidase, which may be ascribed to the interactions between the polysaccharide and gut microbiota.

Previous studies reported the effects of several S. fusiforme polysaccharides on gut microbiota composition $[11,17,33]$. Chen et al. reported that 12 months of oral administration of an S. fusiforme polysaccharide decreased the abundance of the phyla Firmicutes, Proteobacteria, and the genera Lactobacillus and Helicobacter in small intestinal microbiota [33]. Cheng and colleagues found that 6 weeks of oral administration of an S. fusiforme polysaccharide significantly decreased the relative abundances of several diabetes-related mi- 
crobiota in fecal samples, including Bilophila, Oscillibacter, and Mucispirillum [17]. Our previous study reported that 4 weeks of $S$. fusiforme polysaccharide treatment significantly altered the relative abundance of the phylum Bacteroidetes, and the genera Oscillospira, Mucispirillum, and Clostridiales in cecal microbiota [11]. In the present study, 16 weeks of SfW administration partially reversed HFD-induced alterations of Clostridiales and $R u$ minococcaceae in both the cecal and fecal microbiota. The differences in the regulatory effects of S. fusiforme polysaccharides on gut micobiota may be explained by the structural difference of polysaccharides, or difference in the animal model, and sampling position. For example, the microbiota composition in the intestinal contents from the ICR mice was investigated by Chen et al., while in the present study, we determined the microbiota composition in fecal and cecal samples from the ICR mice.

\section{Materials and Methods}

\subsection{Materials}

The brown algae S. fusiforme was sourced from Qingdao, China, on August, 2018. Standards of monosaccharides and 1-phenyl-3-methyl-5-pyrazolone (PMP) were purchased from Aladdin Chemistry Co., Ltd. (Shanghai, China). Dextran standards were purchased from American Polymer Standards Corporation (Mentor, OH, USA). Other reagents and solvents were of analytical grade.

\subsection{Preparation of Sargassum fusiforme Polysaccharides}

As previously reported, S. fusiforme polysaccharides were prepared with slight modifications [11]. Briefly, dry S. fusiforme was cut into pieces and pre-treated with $95 \%$ ethanol to remove the pigment. Polysaccharides were extracted from the residual materials by hot water for $1 \mathrm{~h}$ and $0.1 \mathrm{M} \mathrm{HCl}$ at $60^{\circ} \mathrm{C}$ for $1 \mathrm{~h}$, respectively. The hot water-extracted polysaccharide was further treated with $0.05 \mathrm{M} \mathrm{MgCl}_{2}$ to eliminate the alginate, and ultra-filtered to obtain the SfW. The $\mathrm{HCl}$-extracted crude polysaccharide was dialyzed and precipitated using ethanol to obtain the SfA.

\subsection{Structural Analysis of Sargassum fusiforme Polysaccharides}

The chemical analysis of SfW and SfA was based on previous studies [11]. The total sugar content was measured by the phenol-sulfuric acid method using D-glucose as the standard [34]. The content of sulfate was analyzed with the $\mathrm{BaCl}_{2}$-gelation method using $\mathrm{Na}_{2} \mathrm{SO}_{4}$ as the standard [35]. The molecular weight analysis was conducted using HighPerformance Size Exclusion Chromatography using a Waters 2487 HPLC system with a refractive index detector 2414 (Waters, Milford, MA, USA). The chromatography conditions refer to previous studies [11]. The molar ratio of monosaccharide and fucose content was determined by the PMP derivatization method with minor modification.

SfW and SfA fraction (100 mg) were dissolved in $0.55 \mathrm{~mL}$ of $\mathrm{D}_{2} \mathrm{O}(99.9 \%)$ followed by centrifugation and lyophilization, and the process was repeated three times. Finally, the resulting polysaccharide was dissolved with $0.55 \mathrm{~mL}$ of $\mathrm{D}_{2} \mathrm{O} .{ }^{1} \mathrm{H}$ NMR spectra were recorded on AVANCE III NMR $600 \mathrm{MHz}$ spectrometer (Bruker Inc., Billerica, MA, USA) at $25^{\circ} \mathrm{C}$.

\subsection{Animal Experiments}

Twenty-two male C57 mice (Specific-pathogen-free grade, six-week-old) were purchased from SPF (Beijing, China) Biotechnology Co., Ltd. (Beijing, China), and kept at the Animal Center of Zhejiang, University of Technology. All mice were randomly divided into four groups (the blank and control groups: $n=6$; the SfW and SfA groups: $n=5$ ) and fed a normal diet for one week to stabilize all the metabolic conditions. Each group was housed in one standard cage under a condition of $22 \pm 1{ }^{\circ} \mathrm{C}$, a humidity of $55 \pm 5 \%$, and a $12 \mathrm{~h}$ light/dark cycle. From the beginning of the experiment, mice in the control, SfW, and SfA groups were fed with an HFD (TP23300, $60 \mathrm{kcal} \%$ fat, Trophic Animal Feed High-Tech Co., Ltd, Nantong, China), and mice in the blank group were still fed a standard lab chow 
diet. The mice in the SfW and SfA groups had free access to $1 \mathrm{mg} / \mathrm{mL}$ of SfW and SfA in drinking water, respectively, for a period of 16 weeks, and the other two groups were treated with sterile water.

During the experiments, the body weight was measured weekly, and fasting blood glucose was measured monthly via tail vein using a glucometer (Johnson and Johnson, New Brunswick, NJ, USA) according to the instruction after fasting for $16 \mathrm{~h}$. A sugar tolerance test (OGTT) and insulin tolerance test (ITT) were measured at week 15 and 16, respectively, and the feces were collected before the mice were dissected. All mice were sacrificed from asphyxiation by carbon dioxide. The liver and epididymal fat were harvested and their weight was measured. The cecum contents were collected and stored at $-80{ }^{\circ} \mathrm{C}$. The protocol was approved by the Animal Ethics Committee of the Zhejiang University of Technology, China. All efforts were made to minimize the suffering of the mice.

\subsection{Gut Microbiota Analysis by $16 \mathrm{~S}$ rRNA Gene Sequencing}

Fecal samples and cecal contents were used for gut microbiota analysis by sequencing the $16 \mathrm{~S}$ rRNA genes. The DNA extraction, PCR amplification, sequencing, and data analysis were conducted according to our previous study [11].

\subsection{Statistical Analysis}

The significance of the differences between the two groups was assessed using the unpaired two-tailed Student's $t$-test (Figure 1). Data sets that involved more than two groups were assessed by one-way ANOVA followed by a Turkey's test (Figures 1-4). $p$ values and the significance level are indicated in the associated figure legends for each figure. Statistical analysis was performed with SPSS statistics software (Version 19.0).

\section{Conclusions}

In conclusion, we compared the microbiota composition in the cecal and fecal microbiota of 16-week HFD-fed mice and revealed that HFD dramatically altered the abundance of Bacteroidetes and Actinobacteria, and Coriobacteriaceae, S24-7, and Ruminococcus in both the cecal and fecal microbiota but did not affect the relative abundance of Firmicutes, Clostridiales, Oscillospira, Ruminococcaceae in cecal microbiota and the Simpson's index of fecal microbiota. Co-treatments with SfW and SfA exacerbate body weight gain and partially reverse HFD-induced alterations of Clostridiales and Ruminococcaceae and alter the abundance of genes encoding monosaccharide-transporting ATPase, $\alpha$-galactosidase, $\beta$-fructofuranosidase, and $\beta$-glucosidase. Our findings provide important insights for the study of seaweed polysaccharide-based gut microbiota regulators.

Supplementary Materials: The following are available online at https://www.mdpi.com/article/ 10.3390/md19070364/s1. Figure S1 ${ }^{1} \mathrm{H}-\mathrm{NMR}$ spectra of Sargassum fusiforme polysaccharides SfW and SfA.

Author Contributions: X.-W.X. and H.W. designed the study; B.W.(Bin Wei), Q.-L.X., B.Z., T.-S.Z., and S.-Z.K. performed the animal study; S.J.W. characterized the structures of polysaccharides; B.W.(Bin Wei) and Q.-L.X. wrote the manuscript; B.W.(Bin Wu) revised the manuscript; X.-W.X. and H.W. supervised the study. All authors have read and agreed to the published version of the manuscript.

Funding: This work was financially supported by the National Key Research and Development Program (No. 2017YFE0103100) and the programs of the National Natural Science Foundation of China (No. 81903534, No. 81773628, and No. 81741165) and the Scientific Research Fund of Second Institute of Oceanography, MNR (No. JB2002).

Institutional Review Board Statement: The study was conducted according to the guidelines of the Declaration of Helsinki, and approved by the Ethics Committee of the Zhejiang University of Technology, China (No. 20190219043).

Data Availability Statement: The data in this study are available on request from the corresponding author. 
Conflicts of Interest: There are no conflict to declare.

\section{References}

1. Valdes, A.M.; Walter, L.; Segal, E.; Spector, T.D. Role of the gut microbiota in nutrition and health. BMJ Brit. Med. J. 2018, 361, j2179. [CrossRef] [PubMed]

2. Chassaing, B.; Koren, O.; Goodrich, J.K.; Poole, A.C.; Srinivasan, S.; Ley, R.E.; Gewirtz, A.T. Dietary emulsifiers impact the mouse gut microbiota promoting colitis and metabolic syndrome. Nature 2015, 519, U92-U192. [CrossRef] [PubMed]

3. Stephens, R.W.; Arhire, L.; Covasa, M. Gut microbiota: From microorganisms to metabolic organ influencing obesity. Obesity 2018, 26, 801-809. [CrossRef] [PubMed]

4. Hills, R.D.; Pontefract, B.A.; Mishcon, H.R.; Black, C.A.; Sutton, S.C.; Theberge, C.R. Gut microbiome: Profound implications for diet and disease. Nutrients 2019, 11, 1613. [CrossRef]

5. Yu, F.; Han, W.; Zhan, G.F.; Li, S.; Jiang, X.H.; Wang, L.; Xiang, S.K.; Zhu, B.; Yang, L.; Luo, A.L.; et al. Abnormal gut microbiota composition contributes to the development of type 2 diabetes mellitus in db/db mice. Aging 2019, 11, 10454-10467. [CrossRef]

6. Nguyen, S.G.; Kim, J.; Guevarra, R.B.; Lee, J.H.; Kim, E.; Kim, S.I.; Unno, T. Laminarin favorably modulates gut microbiota in mice fed a high-fat diet. Food Funct. 2016, 7, 4193-4201. [CrossRef]

7. Marteau, P.; Pochart, P.; Dore, J.; Bera-Maillet, C.; Bernalier, A.; Corthier, G. Comparative study of bacterial groups within the human cecal and fecal microbiota. Appl. Environ. Microb. 2001, 67, 4939-4942. [CrossRef]

8. Stanley, D.; Geier, M.S.; Chen, H.; Hughes, R.J.; Moore, R.J. Comparison of fecal and cecal microbiotas reveals qualitative similarities but quantitative differences. BMC Microbiol. 2015, 15, 1-11. [CrossRef]

9. Guo, B.B.; Yang, B.; Pang, X.Y.; Chen, T.P.; Chen, F.; Cheng, K. Fucoxanthin modulates cecal and fecal microbiota differently based on diet. Food Funct. 2019, 10, 5644-5655. [CrossRef]

10. Shang, Q.S.; Song, G.R.; Zhang, M.F.; Shi, J.J.; Xu, C.Y.; Hao, J.J.; Li, G.Y.; Yu, G.L. Dietary fucoidan improves metabolic syndrome in association with increased Akkermansia population in the gut microbiota of high-fat diet-fed mice. J. Funct. Foods 2017, 28 138-146. [CrossRef]

11. Li, S.; Li, J.H.; Mao, G.Z.; Wu, T.T.; Hu, Y.Q.; Ye, X.Q.; Tian, D.; Linhardt, R.J.; Chen, S.G. A fucoidan from sea cucumber Pearsonothuria graeffei with well-repeated structure alleviates gut microbiota dysbiosis and metabolic syndromes in HFD-fed mice. Food Funct. 2018, 9, 1039. [CrossRef]

12. You, L.; Gong, Y.; Li, L.; Hu, X.; Brennan, C.; Kulikouskaya, V. Beneficial effects of three brown seaweed polysaccharides on gut microbiota and their structural characteristics: An overview. Int. J. Food Sci. Technol. 2020, 55, 1199-1206. [CrossRef]

13. Zhang, R.; Zhang, X.; Tang, Y.; Mao, J. Composition, isolation, purification and biological activities of Sargassum fusiforme polysaccharides: A review. Carbohydr. Polym. 2020, 228, 115381. [CrossRef]

14. Kong, Q.; Zhang, R.; You, L.; Ma, Y.; Liao, L.; Pedisić, S. In vitro fermentation characteristics of polysaccharide from Sargassum fusiforme and its modulation effects on gut microbiota. Food Chem. Toxicol. 2021, 151, 112145. [CrossRef]

15. Zhang, Y.; Zuo, J.; Yan, L.; Cheng, Y.; Li, Q.; Wu, S.; Chen, L.; Thring, R.W.; Yang, Y.; Gao, Y.; et al. Sargassum fusiforme fucoidan alleviates high-fat diet-induced obesity and insulin resistance associated with the improvement of hepatic oxidative stress and gut microbiota profile. J. Agric. Food Chem. 2020, 68, 10626-10638. [CrossRef]

16. Wei, B.; Zhong, Q.W.; Ke, S.Z.; Zhou, T.S.; Xu, Q.L.; Wang, S.J.; Chen, J.W.; Zhang, H.W.; Jin, W.H.; Wang, H. Sargassum fusiforme polysaccharides prevent high-fat diet-induced early fasting hypoglycemia and regulate the gut microbiota composition. Mar. Drugs 2020, 18, 444. [CrossRef]

17. Cheng, Y.; Sibusiso, L.; Hou, L.F.; Jiang, H.J.; Chen, P.C.; Zhang, X.; Wu, M.J.; Tong, H.B. Sargassum fusiforme fucoidan modifies the gut microbiota during alleviation of streptozotocin-induced hyperglycemia in mice. Int. J. Biol. Macromol. 2019, 131, 1162-1170. [CrossRef]

18. Palanisamy, S.; Vinosha, M.; Marudhupandi, T.; Rajasekar, P.; Prabhu, N.M. Isolation of fucoidan from Sargassum polycystum brown algae: Structural characterization, in vitro antioxidant and anticancer activity. Int. J. Biol. Macromol. 2017, 102, 405-412. [CrossRef]

19. Somasundaram, S.N.; Shanmugam, S.; Subramanian, B.; Jaganathan, R. Cytotoxic effect of fucoidan extracted from Sargassum cinereum on colon cancer cell line HCT-15. Int. J. Biol. Macromol. 2016, 91, 1215-1223. [CrossRef]

20. Synytsya, A.; Kim, W.J.; Kim, S.M.; Pohl, R.; Synytsya, A.; Kvasnička, F.; Čopíková, J.; Park, Y. Structure and antitumour activity of fucoidan isolated from sporophyll of Korean brown seaweed Undaria pinnatifida. Carbohydr. Polym. 2010, 81, 41-48. [CrossRef]

21. Zhong, Q.W.; Zhou, T.S.; Qiu, W.H.; Wang, Y.K.; Xu, Q.L.; Ke, S.Z.; Wang, S.J.; Jin, W.H.; Chen, J.W.; Zhang, H.W.; et al Characterization and hypoglycemic effects of sulfated polysaccharides derived from brown seaweed Undaria pinnatifida. Food Chem. 2021, 341, 128148. [CrossRef]

22. Zhong, Q.W.; Wei, B.; Wang, S.J.; Ke, S.Z.; Chen, J.W.; Zhang, H.W.; Wang, H. The antioxidant activity of polysaccharides derived from marine organisms: An overview. Mar. Drugs 2019, 17, 672. [CrossRef]

23. Kim, M.J.; Jeon, J.; Lee, J.S. Fucoidan prevents high-fat diet-induced obesity in animals by suppression of fat accumulation. Phytother. Res. 2014, 28, 137-143. [CrossRef]

24. Fitton, J.H.; Stringer, D.N.; Karpiniec, S.S. Therapies from fucoidan: An update. Mar. Drugs 2015, 13, 5920-5946. [CrossRef]

25. Fitton, J.H. Therapies from fucoidan; multifunctional marine polymers. Mar. Drugs 2011, 9, 1731-1760. [CrossRef]

26. Li, B.; Lu, F.; Wei, X.J.; Zhao, R.X. Fucoidan: Structure and bioactivity. Molecules 2008, 13, 1671-1695. [CrossRef] 
27. Agus, A.; Clément, K.; Sokol, H. Gut microbiota-derived metabolites as central regulators in metabolic disorders. Gut 2020, 70, 1174-1182. [CrossRef]

28. Ding, N.; Zhang, X.; Zhang, X.D.; Jing, J.; Zhao, A.Z. Impairment of spermatogenesis and sperm motility by the high-fat diet-induced dysbiosis of gut microbes. Gut 2020, 69, 1608-1619. [CrossRef] [PubMed]

29. Shin, N.R.; Lee, J.C.; Lee, H.Y.; Kim, M.S.; Whon, T.W.; Lee, M.S.; Bae, J.W. An increase in the Akkermansia spp. population induced by metformin treatment improves glucose homeostasis in diet-induced obese mice. Gut 2014, 63, 727-735. [CrossRef] [PubMed]

30. Alessandro, T.; Valeria, M.; Cristina, F.; Antonio, P.; Marcello, A.; Massimo, D.; Michael, S.; Sergio, U. Metaproteogenomics reveals taxonomic and functional changes between cecal and fecal microbiota in mouse. Front. Microbiol. 2017, 8, 391.

31. Shahinozzaman, M.; Raychaudhuri, S.; Fan, S.; Obanda, D.N. Kale attenuates inflammation and modulates gut microbial composition and function in C57BL/6J mice with diet-induced obesity. Microorganisms 2021, 9, 238. [CrossRef]

32. Kozik, A.J.; Nakatsu, C.H.; Chun, H.; Jones-Hall, Y.L. Comparison of the fecal, cecal, and mucus microbiome in male and female mice after TNBS-induced colitis. PLoS ONE 2019, 14, e0225079. [CrossRef]

33. Chen, P.; Yang, S.; Hu, C.; Zhao, Z.; Wu, M. Sargassum fusiforme polysaccharide rejuvenates the small intestine in mice through altering its physiology and gut microbiota composition. Curr. Mol. Med. 2017, 17, 350-358.

34. Lin, F.M.; Pomeranz, Y. Effect of borate on colorimetric determinations of carbohydrates by the phenol-sulfuric acid method. Anal. Biochem. 1968, 24, 128-131. [CrossRef]

35. Dodgson, K.; Price, R. A note on the determination of the ester sulphate content of sulphated polysaccharides. Biochem. J. 1962, 84, 106-110. [CrossRef] 